\title{
Spatiotemporal Decomposition in Object-Space along Reconstruction in Emission Tomography
}

\author{
Xavier Hubert ${ }^{1,3}$, Dominique Chambellan ${ }^{1}$, Samuel Legoupil ${ }^{1}$, \\ Régine Trébossen ${ }^{2}$, Jean-Robert Deverre ${ }^{2}$, and Nikos Paragios ${ }^{3,4}$ \\ ${ }^{1}$ CEA, LIST, Laboratoire Images et Dynamique, Gif/Yvette, F-91191, France \\ xavier.hubert@cea.fr \\ ${ }^{2}$ CEA, I2BM, Orsay, F-91400, France \\ ${ }^{3}$ Laboratoire MAS, Ecole Centrale de Paris, France \\ ${ }^{4}$ Equipe GALEN, INRIA-Saclay, Ile-de-France, France
}

\begin{abstract}
Emission tomography has provided a new insight in brain mechanisms past years. Although reconstructions are nowadays mostly static, trend is going toward dynamic acquisitions and reconstructions. This opens a new range of investigations, for instance for drugs discovery. Indeed new drugs are studied through the dynamic ability of tissues to catch them. However, it is required to know radiotracer concentration of blood that irrigates tissues in order to draw conclusions on potentials of these drugs. This concentration is called 'input function' and this paper presents a new method for measuring it in a non-invasive way.

Our new method relies on simultaneous estimations of vessels kinetics and vessels spatial distribution. These estimations are performed during the reconstruction process and take into account the statistical nature of measured signals. Indeed, this method is based on the maximisation of the likelihood of counts in detectors. It takes advantages of a nonnegative matrix factorisation which separate spatial and temporal components. Results are very promising, since it estimates arterial input function accurately although object emits just a limited amount of photons, especially within the first minutes.
\end{abstract}

\section{Introduction}

Emission-tomography is getting more and more useful for medical applications, in particular for quantification of physiological parameters for drug discovery. Depending on radioisotopes that label molecules, PET or SPECT enable the reconstruction of $3 \mathrm{D}$ radioactive spatial distributions. Such scanners provide dynamic sequences of images and consequently dynamic information on physiological parameters. In this context, measurements along time of radiotracer concentrations in arterial blood allow the derivation of some important information on kinetics of labelled molecules [1, by comparing them to uptake values in organs. Non-invasive measurements of this concentration, which is called the input function, have strong clinical interest, but is difficult to achieve because of low amount of photons which are emitted from vessels. Furthermore, veins 
are always located close to arteries. This implies that spatial resolution has to be smaller than characteristic dimensions of vessels, which is about a few millimetres. Consequently it is very relevant to improve dynamic reconstruction methods in order to quantify concentrations of radiotracers flooding in arteries.

The state-of-the-art technique relies on arterial blood sampling [2, which is an invasive and painful procedure for patients. Moreover medical staff is exposed to unnecessary radiation. This method requires drawing samples at pre-established time points, which has the disadvantage to induce uncertainty on the exact moment when the input function reaches its maximum as well as its maximum value. These two parameters are very relevant for pharmaco-kinetics studies. It has been proposed to extract directly the input function from PET reconstructions [3] through independent component analysis. This method depends on the reconstructed spatial distribution. While every time frame is reconstructed independently, first frames are noisy and consequently estimation of kinetics is not accurate. Some recent works propose to include the kinetics estimations in reconstruction process [4 [5], but these methods require to provide functions basis to estimate kinetics, which is a strong prior. It has also been suggested to perform a non-negative matrix factorisation (NMF) of the dynamic projection data in order to separate spatial and temporal information. Iterative algorithms [6] [7], as well as projected gradient methods [8], have been proposed, but NMF is then performed in the projection space. Thus performances of these methods depend strongly on the reconstruction quality of every time frame.

As an alternative to these methods, we propose to perform NMF in the object space. Indeed, components are expected to be spatially separated in object space, whereas contributions of components in projections can be mixed up together. Performing NMF in object space requires that it is introduced in the reconstruction process. Our new algorithm estimates simultaneously kinetics in the object and its spatial distribution. This method reconstructs 4D-objects from just two projections that are acquired through coded-aperture collimators, with the assumptions that the object is still and the kinetics is homogeneous inside every vessel. It is based on the maximisation of Poisson likelihood. Consequently, it is an appropriate statistical model for projections with low counts in pixels. Upon improvement on the collimation procedure, SPECT can be a good solution for measuring the input function. Sensitivity of imaging system can be significantly augmented by using a coded-aperture collimator with a gamma-camera or a gamma-imager [9]. In this case, it is possible to use SPECT for problems dealing with low available amount of information, as non-invasive determination of input function. In particular it is well suited for imaging objects emitting photons with middle and high energy which are more difficult to collimate and to detect.

\section{Method}

Our aim is to reconstruct vessels, at locations such as the knees, which stand far from main active organs (brain, heart, bladder). This reduces external noise in projections. Furthermore knees are of particular interest since arteries are almost 

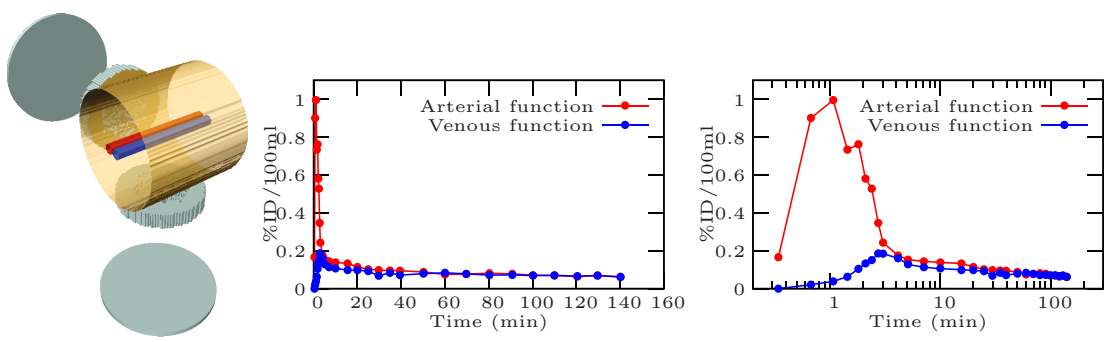

Fig. 1. (Left) Imaging configuration with two detectors and two collimators (Centre) Ground-truth kinetics (Right) Ground-truth kinetics in log-scale representations

as large as the femoral artery, but closer to the surface, which is important for detection efficiency. We test our method through simulations and experiments.

The configuration of our imaging system relies on two $\gamma$-imagers. The scintillator of every detector is made of CsI. It is $4 \mathrm{~mm}$-thick and its diameter is 100 mm-large. Such detectors produce $(128 \times 128)$-pixel images. Collimators are coded apertures of type HURA [10. They are $9 \mathrm{~mm}$-thick and centre-to-centre hole distance is $1,85 \mathrm{~mm}$. Collimators are made of tungsten. Artery and vein are assumed to be cylindrical. For both simulations and experiments, we model them as $5 \mathrm{~mm}$-large and $30 \mathrm{~mm}$-long cylinders. They emit $511 \mathrm{keV}$-photons. Imaging configuration, which have been tested through simulations and experiments, is described in Fig 1. The axis-to-axis distance of cylinders is $8 \mathrm{~mm}$. Cylinders stand in the centre of the knee which is 100 mm-large. Collimators are in contact with the knee and detectors stand 100 mm-away from collimators. Kinetics in vessels that are presented in Fig 1 have been obtained through arterial and venous blood samplings. Artery and vein kinetics only differ in the first 7-8 minutes, which means that temporal resolution must be excellent during the first minutes. In this figure, $\% I D / 100 \mathrm{ml}$ stands for 'percentage of injected dose per 100 millilitres'. The injected dose is $5 \mathrm{mCi}$. From these kinetics, projections on detectors have first been generated through simulations for every time frame with Monte-Carlo code GATE [11. Real data acquisitions have been then performed on a real gamma-imager. As we just have one imager, we rotate the object in order to acquire two orthogonal projections [12].

\section{NMF in Object-Space Based on Maximisation of Likelihood of Poisson Signals}

\subsection{Model}

Dynamic imaging is based on the acquisition of a set of projections $S_{t}$ for every time frame $t$. During every time frame $t$, an object $O$ emits photons and their $3 \mathrm{D}$ spatial distribution on a voxel grid is denoted $\lambda_{t}$. The number of voxels is denoted $M . \lambda_{t}$ is a $M$-long $3 \mathrm{D}$-vector that is reorganised as a $1 \mathrm{D}$-vector for computational purpose. We then denoted $a_{i, j}$ the probability that a photon which 
has been emitted from voxel $j$ is detected in pixel $i$ of a set of detectors $D$. All $a_{i, j}$ constitutes a matrix $A$ that models the imaging system. $A$ does not depend on the time frame as long as the imaging system and the object are still. It can be noticed that every pixel count $S_{t}(i)$ is a draw of a Poisson-distributed variable whose mean is $\left(A \lambda_{t}\right)(i)$. Reconstructing the object $O$ from the set of projections $S_{t}$ consists in estimating the $3 \mathrm{D}$ spatial distribution $\bar{\lambda}_{t}$ that minimises the Poisson noise $n$ given by: $S_{t}=A \cdot \lambda_{t}+n$. All time frames are acquired independently, as a result all $S_{t}$ are statistically independent one from another. Let define $S$ (respectively $\Lambda$ ) as the matrix whose columns are the vectors $S_{t}$ (respectively the vectors $\lambda_{t}$ ). Reconstructing all time frames simultaneously can be performed by estimating the matrix $\bar{\Lambda}$ that minimises the Poisson noise $N_{\Lambda}$ :

$$
S=A \cdot \Lambda+N_{\Lambda}
$$

This means that the number of variables $\Lambda_{j, t}$ in the problem increases linearly with the number of frames. The better the time resolution, the larger the number of variables $\Lambda_{j, t}$ to estimate. Furthermore, the amount of detected photons in every time frame is usually not sufficient to reconstruct 3D-distribution of the object, although this step is required to define region of interests (ROI) in which we are interested to know the activity in order to assess the input function. As an alternative, we propose to look for a non-negative matrix factorisation of $\Lambda$ which deconvolves spatial information from kinetics information. Indeed, for every time frame $t, \lambda_{t}$ can be viewed as the sum of the contributions of the artery and the vein, that is to say: $\lambda_{t}=\lambda_{t}^{a}+\lambda_{t}^{v}$. By assuming that the kinetics is homogeneous in every cylinder, we can write that : $\lambda_{t}^{a}=K_{t}^{a} \cdot\left[X_{1}^{a} \ldots X_{M}^{a}\right]^{T}+n_{t}^{a}$ and $\lambda_{t}^{v}=$ $K_{t}^{v} \cdot\left[X_{1}^{v} \ldots X_{M}^{v}\right]^{T}+n_{t}^{v}$, where $\left(X^{a}\right)^{T}$ and $\left(X^{v}\right)^{T}$ describe the transposes of the spatial locations of the artery and the vein. They do not depend on time frames. $K_{t}^{a}$ and $K_{t}^{v}$ are the kinetics of the artery and the vein for time frame $t . n_{t}^{a}$ and $n_{t}^{v}$ are Poisson noise. We assume that there are $T$ time frames. Object $O$ is made of $M$ voxels. The $4 \mathrm{D}$ activity distribution $\Lambda$ can then be written as:

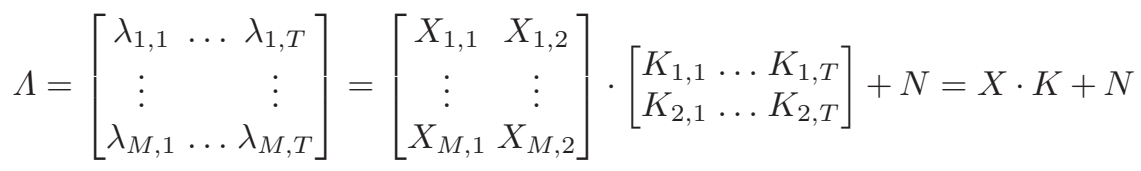

where $N$ is a Poisson noise, $X$ descibes spatial distribution of components and $K$ describes their kinetics. $\left(X_{i}^{a}, X_{i}^{v}, K_{j}^{a}, K_{j}^{v}\right)$ has been replaced for all $i, j$ by $\left(X_{i, 1}, X_{i, 2}, K_{1, j}, K_{2, j}\right)$ for clarity in next analytical calculations. Factorisation is a valid model as long as the following assumptions are satisfied: (i) kinetics of artery and vein are homogeneous in each cylinder, and (ii) the object does not move during the acquisition. The model involves two components, because we expect to observe measurements due to the artery and the vein.

Before the factorisation, $M \times T$ parameters have to be estimated whereas after the factorisation on two components, it is reduced to $2 \times M+2 \times T \sim 2 \times M$, since usually the number of voxels $M$ is far larger than the number of time frames $T$. Consequently, after factorisation, there are about $T / 2$ less parameters 
to estimate. This is significant since there are usually several dozens of time frames per acquisition. The point is that $X$ and $K$ are estimated instead of A. Furthermore, spatial matrix $X$ is estimated from all the time frames, which reduces the problems of low counts in first frames. We are now looking for the couple $\{X ; K\}$ that minimises the Poisson noise $N^{\prime}$ in:

$$
S=A X K+N^{\prime}
$$

This is done through the maximisation of the likelihood $L(X, K)$ which is proportional to the conditional probability $P(S \mid X, K)$ :

$$
L(X, K) \propto \prod_{i, j} \frac{(A X K)_{i, j}^{S_{i, j}} e^{-(A X K)_{i, j}}}{S_{i, j} !}
$$

\subsection{Cost Function and Gradient Derivation}

For this problem, it is very important to perform an optimisation which is based on likelihood of a Poisson mixture because the number of detected photons in detectors is low, which does not allow approximation of Poisson variables by Gaussian variables. Maximising the likelihood $L(X, K)$ is equivalent to maximising its modified logarithm:

$$
E=\ln (L(X, K))+\ln \left(\prod_{i, j}\left(S_{i, j} !\right)\right)=\sum_{i, j}\left[S_{i, j} \ln \left((A X K)_{i, j}\right)-(A X K)_{i, j}\right]
$$

The number of components is denoted $C$. We remember that the object is made of $M$ voxels and that there are $T$ time frames. Differentiations are calculated analytically from Eq. 5 .

$$
\begin{aligned}
& \forall(p, q, t) \in \llbracket 1 ; M \rrbracket \times \llbracket 1 ; C \rrbracket \times \llbracket 1 ; T \rrbracket \\
& \frac{\partial E}{\partial X_{p, q}}=\sum_{i, j} A_{i, p}\left(\frac{S_{i j}}{(A X K)_{i j}}-1\right) K_{q, j}=\left(A^{T}\left(\frac{S}{A X K}-1\right) K^{T}\right)_{p, q} \\
& \frac{\partial E}{\partial K_{q, t}}=\sum_{i, k} X_{k, q} A_{i, k}\left(\frac{S_{i, t}}{(A X K)_{i, t}}-1\right)=\left(X^{T} A^{T}\left(\frac{S}{A X K}-1\right)\right)_{q, t}
\end{aligned}
$$

We then use a constraint spectral projected gradient method [13] to maximise the modified log-likelihood $E$ on positive sets. From Eq. 6] and 7, it is observed that optimisation is not convex and consequently, initialisation step must be dealt with carefully.

\subsection{Initialisation}

The assumption is made that two components stand inside the object. Eq. 7 becomes:

$$
\frac{\partial E}{\partial K_{q, t}}=\sum_{i}\left(\frac{S_{i, t}(A X)_{i, q}}{(A X)_{i, 1} K_{1, t}+(A X)_{i, 2} K_{2, t}}\right)-\sum_{i}(A X)_{i, q}
$$


A rough assumption needs to be done to get a correct initialisation point. We assume that $X$ is made of uniform components, that is to say that every coefficient of $X$ equals an arbitrarily value $\alpha$. Then, a relation is derived between every $K_{1, t}$ and $K_{2, t}$ from Eq. 8 .

$$
\begin{aligned}
\frac{\partial E}{\partial K_{1, t}}=0 \quad & \Leftrightarrow \quad \sum_{i}\left(\frac{\alpha S_{i, t} \sum_{k} A_{i, k}}{\left(\alpha K_{1, t}+\alpha K_{2, t}\right) \sum_{k} A_{i, k}}\right)=\alpha \sum_{i, k} A_{i, k} \\
& \Leftrightarrow \quad K_{1, t}+K_{2, t}=\frac{\sum_{i} S_{i, t}}{\alpha \sum_{i, k} A_{i, k}}
\end{aligned}
$$

Differentiation on $K_{2, t}$ would also lead to Eq. 10. As we expect from physiological information that concentration is always higher in the artery than in the vein, we can also assume that $K_{1, t} \leq K_{2, t}$, that is to say $\exists R_{t} \in[0,1]$ so that $K_{2, t}=R_{t} K_{1, t}$. Initialisation is then performed by choosing a random variable $R_{t}$ from a uniform distribution over $[0 ; 1]$, which leads through Eq. 10 to:

$$
K_{1, t}=\frac{1}{\alpha\left(1+R_{t}\right)} \frac{\sum_{i} S_{i, t}}{\sum_{i, k} A_{i, k}} \text { and } K_{2, t}=\frac{R_{t}}{\alpha\left(1+R_{t}\right)} \frac{\sum_{i} S_{i, t}}{\sum_{i, k} A_{i, k}}
$$

The initial modified $\log$-likelihood $E$ does not depend on $\alpha$ as it can be observed from Eq. 5 and 10 .

$$
E=\sum_{i, j} S_{i, j} \ln \left(\sum_{k} A_{i, k} \frac{\sum_{i^{\prime}} S_{i^{\prime}, j}}{\sum_{i^{\prime}, k^{\prime}} A_{i^{\prime}, k^{\prime}}}-\sum_{i, j, k} A_{i, k} \frac{\sum_{i^{\prime}} S_{i^{\prime}, j}}{\sum_{i^{\prime}, k^{\prime}} A_{i^{\prime}, k^{\prime}}}\right)
$$

\section{Results}

Data have been first generated with Monte-Carlo code GATE. Fig. 2 presents integrated projections over all temporal frames which have been acquired through coded-apertures according to activities of Fig. 1, Maximum pixel count is 13, which justifies the fact that Poisson likelihood has not been approximated by Gaussian likelihood. Reconstruction has been performed with $\alpha=1$. In Fig. 3. the top line (respectively bottom line) shows results that have been
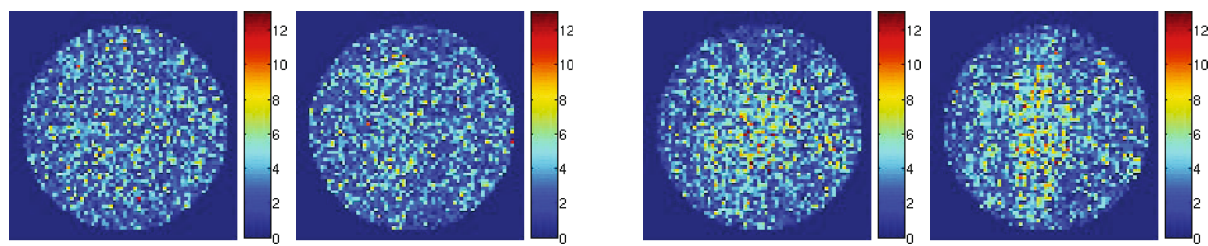

Fig. 2. Integration of all time frames in first and second projections. (Left) GATE simulations: 7868 and 7841 detected photons on the two projections (Right) Experiments : 8590 and 8557 detected photons. 

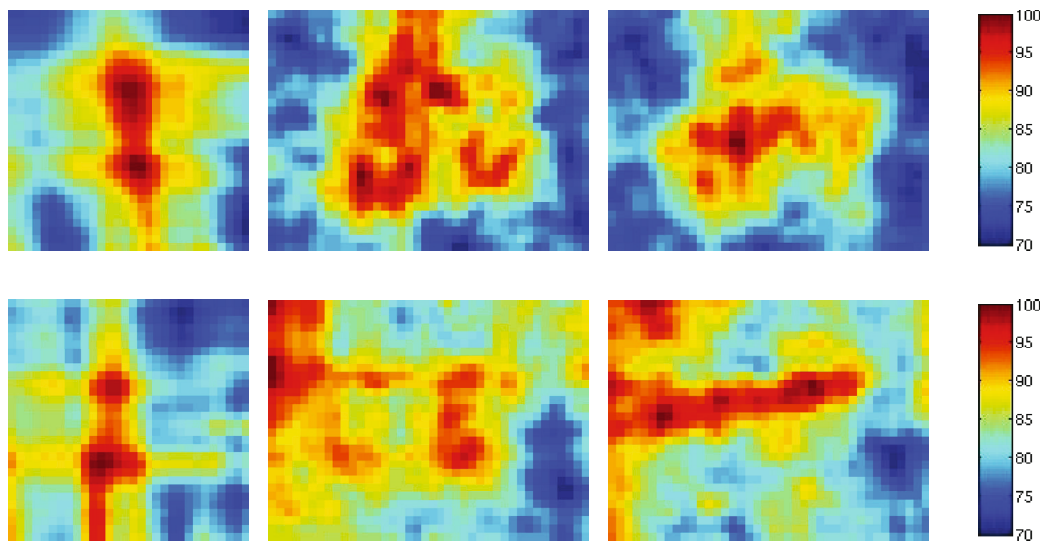

Fig. 3. Normalised integrated views which are perpendicular to the three principal directions: (left) xy-plane (its normal is parallel to cylinders axis), (centre) xy-plane and (right) xz-plane. Reconstructed 3D spatial distribution: (top) Monte Carlo simulations, (bottom) experiments.

obtained through simulations (respectively experiments). Images have been obtained through summation of slices along the three principal directions of reconstructed spatial distribution. From left to right, we can see two spots, which are the sections of both cylinders, then two horizontal cylindrical distribution that stand one above the other, and finally just one because both cylinders stand one behind the other. Numerous artefacts are present in reconstruction. They are due to inaccurate modelling of the transition matrix $A$. Indeed, it has been computed through ray-tracing, which does not take into account all physical phenomena. Whereas spatial components are not clearly distinguishable, components of $K$ estimate accurately the initial kinetics as shown in Fig 4 . The estimation of the fourth time frame in the experimental kinetics is not accurate, which shows that we are close from the limit of the method. In both case, arterial component is better estimated than the venous one. Indeed, it is harder to extract weaker
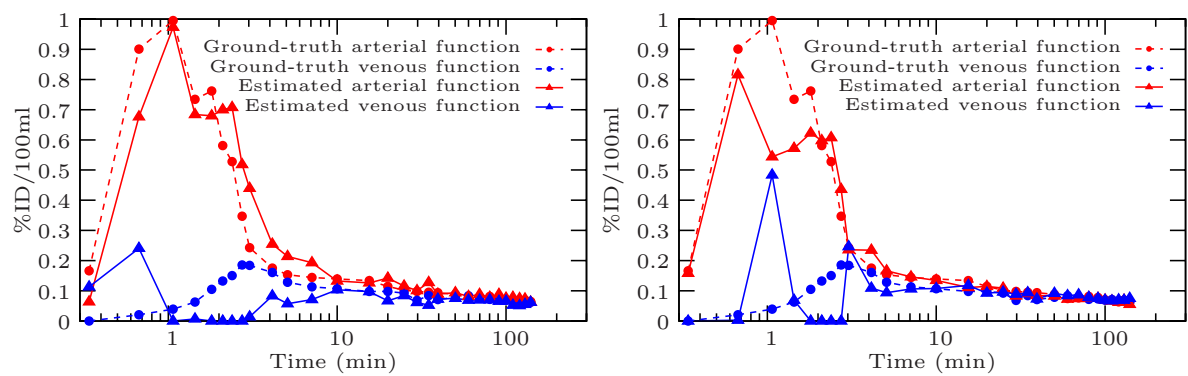

Fig. 4. Estimated kinetics (Left) from simulation data, (Right) from experiments 
signal. This does not affect the goal of this work, which is focused on the input function estimation.

\section{Conclusions}

In this paper we have proposed a novel approach for local reconstruction of PET/SPECT signals. Our method is based on non-negative matrix factorisation. It deeply reduces the number of variables and explicitly encodes the temporal correlation in the reconstruction. Therefore, it can provide reliable estimations even with sparse measurements through appropriate exploitation of Poisson likelihood.

\section{References}

1. Gallezot, J.D., et al.: Quantification of cerebral nicotinic acetylcholine receptors by PET using 2-[(18)F]fluoro-A-85380 and the multiinjection approach. Journal of Cerebral Blood Flow \& Metabolism (2007)

2. Eriksson, L., et al.: Automated blood sampling systems for Positron Emission Tomography. IEEE Trans. Nucl. Sc. 35(1) (1988)

3. Naganawa, M., Kimura, Y., Ishii, K., Oda, K., Ishiwata, K.: Temporal and spatial blood information estimation using Bayesian ICA in dynamic cerebral Positron Emission Tomography. Digital Signal Processing 17(5) (2007)

4. Reader, A., et al.: Joint estimation of dynamic PET images and temporal basis functions using fully 4D ML-EM. Physics in Medicine and Biology 51 (2006)

5. Li, Q., et al.: A fast fully 4-D incremental gradient reconstruction algorithm for list mode PET data. IEEE Trans. Med. Imag. 26(1) (2007)

6. Lee, D.D., Seung, H.S.: Algorithms for Non-negative Matrix Factorization. In: NIPS, pp. 556-562 (2000)

7. Lee, J.S., et al.: Non-negative matrix factorization of dynamic images in nuclear medicine. In: Nucl. Sc. Symp. Conf. Record (2002)

8. Lin, C.J.: Projected gradient methods for Nonnegative Matrix Factorization. Neural Comput. 19(10), 2756-2779 (2007)

9. Cannon, T.M., Fenimore, E.E.: Coded aperture imaging with uniformly redundant arrays. Appl. Opt. 17(3), 337-347 (1978)

10. Finger, M.H., Prince, T.A.: Hexagonal uniformly redundant arrays for codedaperture imaging. In: Proc. 19th Int. Cosmic Ray Conf., pp. 295-298 (1985)

11. Buvat, I., Lazaro, D.: Monte-Carlo simulations in emission tomography with GATE: An overview. N.I.M. in Physics Research A 569(2) (2006)

12. Hubert, X., et al.: Conjoint use of coded-aperture collimators and MLEM algorithm: towards large blood vessels reconstruction at $511 \mathrm{keV}$. In: IEEE Intern. Symp. Biomed. Imag. (2008)

13. Birgin, E.G., Martínez, J.M., Raydan, M.: Nonmonotone spectral projected gradient methods on convex sets. SIAM J. on Optimization 10(4), 1196-1211 (2000) 